\title{
UV (IUE) spectra of the central stars of high latitude planetary nebulae $\mathrm{Hb7}$ and $\mathrm{Sp} 3^{\star}$
}

\author{
G. Gauba ${ }^{1}$, M. Parthasarathy ${ }^{1,2}$, Y. Nakada ${ }^{3,4}$, and T. Fujii ${ }^{3}$ \\ 1 Indian Institute of Astrophysics, Koramangla, Bangalore 560034, India \\ 2 National Astronomical Observatory, 2-21-1 Osawa, Mitaka, Tokyo 181-8588, Japan \\ 3 Institute of Astronomy, School of Science, University of Tokyo, Bunkyo, Tokyo 113-0033, Japan \\ 4 Kiso Observatory, School of Science, University of Tokyo, Mitaka, Kiso, Nagano 397-0101, Japan
}

Received 15 November 2000 / Accepted 23 April 2001

\begin{abstract}
We present an analysis of the UV (IUE) spectra of the central stars of $\mathrm{Hb} 7$ and Sp3. Comparison with the IUE spectrum of the standard star HD 93205 leads to a spectral classification of O3V for these stars, with an

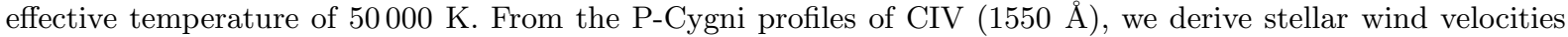
and mass loss rates of $-1317 \mathrm{~km} \mathrm{~s}^{-1} \pm 300 \mathrm{~km} \mathrm{~s}^{-1}$ and $2.9 \times 10^{-8} M_{\odot} \mathrm{yr}^{-1}$ and $-1603 \mathrm{~km} \mathrm{~s}^{-1} \pm 400 \mathrm{~km} \mathrm{~s}^{-1}$ and $7 \times 10^{-9} M_{\odot} \mathrm{yr}^{-1}$ for $\mathrm{Hb} 7$ and Sp3 respectively. From all the available data, we reconstruct the spectral energy distribution of $\mathrm{Hb} 7$ and $\mathrm{Sp} 3$.
\end{abstract}

Key words. planetary Nebulae: individual: Hb7, Sp3 - stars: AGB and Post-AGB - stars: evolution stars: mass-loss - stars: winds - ultraviolet: stars

\section{Introduction}

The central stars of planetary nebulae (CSPNe) are in general very hot objects and their continuum flux is more easily detectable in the UV than in the optical. The detection of fast winds in CSPNe from UV observations may be considered one of the important discoveries of the IUE satellite (Heap 1986; Patriarchi \& Perinotto 1991). NV $(1240 \AA)$ and CIV (1550 $)$ ) resonance line doublets are the most dominant lines formed in the winds of hot stars. A study of the stellar wind profiles of these lines is important to determine the terminal wind velocities and hence the post-AGB mass-loss rate.

We have carried out a program to study the wind profiles of several high galactic latitude planetary nebulae (PN). A monitoring of the NV and CIV wind profiles in Hen 1357 (=SAO 244567) showed wind variability in this young PN (Parthasarathy et al. 1993, 1995) which may be a signature of episodic mass loss in postAGB stars. In this paper we present an analysis of the UV (IUE) low resolution spectra of the high galactic latitude PNe Hb7(PN G003.9-14.9 = IRAS 18523-3219;

Send offprint requests to: G. Gauba,

e-mail: gauba@iiap.ernet.in

* Based on the observations obtained with the International Ultraviolet Explorer (IUE), retrieved from the IUE Final Archive at VILSPA, Madrid, Spain. $l=3.97, b=-14.9)$ and Sp3 (PN G342.5-14.3 = IRAS $18033-5101 ; l=342.51, b=-14.32)$. The photometric colours and optical spectra of these two PNe had indicated that they contain hot central stars (Acker et al. 1992; Aller 1976). We also present the JHK photometry of $\mathrm{Hb} 7$ from the 2MASS Point Source Catalog.

\section{Observations}

Low resolution UV spectra of $\mathrm{Hb} 7$ and $\mathrm{Sp} 3$ were obtained on September 29, 1994 with the SWP camera onboard the IUE satellite. The SWP52257LL image of Hb7 (80 min exposure) and the SWP52256LL image of Sp3 (30 min exposure) were obtained by centering the central stars in the $10^{\prime \prime} \times 23^{\prime \prime}$ aperture. The spectra have been re-extracted from the IUE Final Archive at VILSPA which were reprocessed using the IUE NEWSIPS pipeline which applies the SWET extraction method as well as the latest flux calibration and close-out camera sensitivity corrections. Lineby-line images have been inspected for spurious features.

\section{Analysis}

The spectra of $\mathrm{Hb} 7$ and $\mathrm{Sp} 3$ from $1150 \AA$ to $1950 \AA$ in absolute flux units are shown in Figs. 1a and b. The spectra were dereddened by using $E(B-V)=0.19$ for $\mathrm{Hb} 7$ (from $\mathrm{c}(\mathrm{H} \beta)=0.28$, Tylenda et al. 1989), and $E(B-V)=0.159$ for Sp3 (from HST data, Ciardullo et al. 1999). The dereddened spectra of $\mathrm{Hb} 7$ and $\mathrm{Sp} 3$ were 
compared with the dereddened IUE spectra of standard stars (Heck et al. 1984). For comparison, the three spectra were normalised to $\lambda 1601.53 \AA$. Savage et al. (1985) quote a value of $E(B-V)=0.37$ for the standard $\mathrm{O} 3 \mathrm{~V}$ star, HD93205. Using the $2200 \AA$ feature in the UV, we found $E(B-V)=0.34$ for this star. Hb7 and Sp3 show good agreement with the UV continuum and spectral features of HD93205 (see Fig. 1). Therefore, we adopt the same effective temperature of $50000 \mathrm{~K}$ for the nuclei of $\mathrm{Hb} 7$ and Sp3.

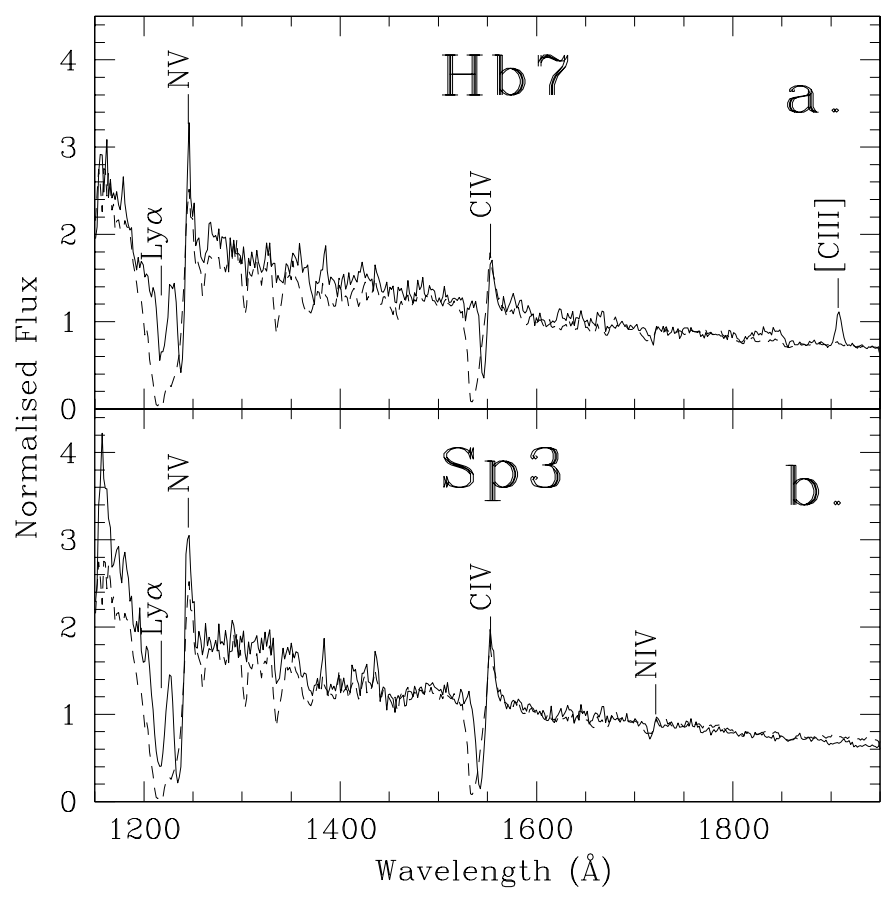

Fig. 1. The dereddened spectra of $\mathrm{Hb} 7 \mathbf{a}), E(B-V)=0.19$ and Sp3 b), $E(B-V)=0.159$ are plotted along with the dereddened SWP spectra of a standard O3-dwarf star (HD 93205, $E(B-V)=0.34)$ from the standard star atlas by Heck et al. (1984). The standard star spectrum is represented by a dotted line.

\subsection{Terminal wind velocity}

Using the violet absorption edge in the high resolution UV spectra of HD 93205, Prinja et al. (1990) calculated a terminal wind velocity $\left(v_{\infty}\right)$ of $-3370 \mathrm{~km} \mathrm{~s}^{-1}$ for this star. They estimated a measurement error of less than $100 \mathrm{~km} \mathrm{~s}^{-1}$ in the determination of this Doppler velocity.

The determination of terminal wind velocity from Doppler shifts in low-dispersion spectra is complicated by the fact that the absorption troughs of strong (saturated) stellar wind lines do not exhibit extended regions of zero residual intensity. In the low resolution spectra of $\mathrm{Hb} 7$ and Sp3, the violet edge of NV is contaminated by Lyman $\alpha$ absorption. By measuring differential shifts of the CIV absorption profiles of the two stars with respect to HD 93205, we found Doppler velocities of $-1435 \mathrm{~km} \mathrm{~s}^{-1}$ for $\mathrm{Hb} 7$ and $-1628 \mathrm{kms}^{-1}$ for $\mathrm{Sp} 3$. These values may be compared with velocities calculated following the analysis of Prinja (1994).

The empirical relation provided by Prinja (1994) uses the difference between the position of the emission peak and the absorption minimum for the CIV line i.e. $v_{\infty}=$ $a_{1}+a_{2}(\Delta \lambda)+a_{3}(\Delta \lambda)^{2}$ where, $a_{1}=-883 \pm 48, a_{2}=$ $259 \pm 9, a_{3}=-3 \pm 2$ and $\Delta \lambda=\lambda_{\text {peak }}^{\text {Emis }}-\lambda_{\text {min }}^{\mathrm{Abs}},(\lambda$ in $\AA$ and $v_{\infty}$ in $\mathrm{kms}^{-1}$ ). Using this relation, we found $v_{\infty}=-1317 \mathrm{~km} \mathrm{~s}^{-1} \pm 316 \mathrm{~km} \mathrm{~s}^{-1}$ for $\mathrm{Hb} 7$ and $v_{\infty}=$ $-1603 \mathrm{~km} \mathrm{~s}^{-1} \pm 389 \mathrm{~km} \mathrm{~s}^{-1}$ for Sp3. Finally, we adopt a terminal velocity of $-1317 \mathrm{~km} \mathrm{~s}^{-1} \pm 300 \mathrm{~km} \mathrm{~s}^{-1}$ for $\mathrm{Hb} 7$ and of $-1603 \mathrm{~km} \mathrm{~s}^{-1} \pm 400 \mathrm{~km} \mathrm{~s}^{-1}$ for Sp3.

\subsection{Stellar temperature and core-mass}

Samland et al. (1992) estimated the temperature of the central star of $\mathrm{Hb} 7$ from photoionization model to be 56000 K. For the central star of Sp3, Preite-Martinez et al. (1991) estimated the energy-balance temperature to be $39400 \mathrm{~K}$. For an O3 star, the effective temperature $\left(T_{\text {eff }}\right)$ is estimated to be $50000 \mathrm{~K}$ (Lang 1992). We adopt the same value of $50000 \mathrm{~K}\left(\log T_{\text {eff }}=4.7\right)$ for the two stars. Pauldrach et al. (1988) analysed the relation between the effective temperature, mass of the nuclei, the terminal velocity and mass-loss rate (see their Figs. 10 and 6a). From these relations, we can deduce a core-mass of 0.644 and $0.565 M_{\odot}$ and a mass-loss rate of $2.9 \times 10^{-8} M_{\odot} \mathrm{yr}^{-1}$ and $7 \times 10^{-9} M_{\odot} \mathrm{yr}^{-1}$ for $\mathrm{Hb} 7$ and Sp3 respectively.

\subsection{Spectral energy distribution (SED)}

The IUE spectra of $\mathrm{Hb} 7$ and Sp3 were combined with the available $B V I$ photometry, $J H K$ photometry (Hb7) from the 2MASS Point Source Catalog and IRAS photometry at $12,25,60$ and $100 \mu \mathrm{m}$ to reconstruct the overall spectral energy distribution (Figs. $2 \mathrm{a}$ and b). The UV data of both stars shows good agreement with a blackbody distribution at $50000 \mathrm{~K}$. The IRAS fluxes do not seem to obey a single black body temperature. By fitting mean blackbody curves to the IRAS fluxes for $\mathrm{Hb} 7$ and Sp3, we estimated cold dust temperatures of $130 \mathrm{~K}$ and $100 \mathrm{~K}$ respectively.

The JHK flux distribution, for $\mathrm{Hb} 7$, shows no indication of the presence of warm dust around the central star. Warm dust is generally attributed to emission from dust grains formed in the outflow close to the central star as a result of on-going post-AGB mass loss. The absence of warm dust may be attributed to photodissociation and diffusion of the dust grains formed close to the hot central star.

Ciardullo et al. (1999) imaged Sp3 with the Wide Field Planetary Camera 2 onboard HST. They found the central star to be a binary with a separation of $0.3^{\prime \prime}$. They found $V=13.20, V-I=-0.19$, and $E(B-V)=0.159$ for the central star and $V=16.86$ and $V-I=0.83$ for the companion. They considered it as a probable physical pair. The binary nature of the nuclei of Sp3 may explain the too bright value of the magnitude calculated by 
Table 1. Photometric data of $\mathrm{Hb} 7$ and $\mathrm{Sp} 3$.

\begin{tabular}{|c|c|c|c|c|c|c|}
\hline & $B$ & $V$ & $I$ & $J$ & $H$ & $K$ \\
\hline $\mathrm{Hb} 7$ & $\begin{array}{l}13.76(\Delta m<0.10) \\
\text { (Tylenda et al. } 1989)\end{array}$ & $\begin{array}{l}13.97(\Delta m<0.10) \\
\text { (Tylenda et al. } 1989)\end{array}$ & - & $\begin{array}{l}12.768 \pm 0.040 \\
(2 \mathrm{MASS}\end{array}$ & $\begin{array}{l}12.866 \pm 0.047 \\
\text { Point Source }\end{array}$ & $\begin{array}{l}12.251 \pm 0.034 \\
\text { Catalog ) }\end{array}$ \\
\hline Sp3 & $\begin{array}{l}12.45(0.10<\Delta m<0.25) \\
\text { (Tylenda et al. } 1991)\end{array}$ & $\begin{array}{l}13.2(\Delta m<0.05) \\
\text { (Ciardullo et al. 1999) }\end{array}$ & $13.39(\Delta m<0.05)$ & - & - & - \\
\hline
\end{tabular}

Tylenda et al. (1991; $B=12.45, V=12.51)$. The $V-I$ colour of the companion is similar to that of an F star. The $B$ magnitude by Tylenda et al. (1991) has been corrected for the contribution from a main sequence $\mathrm{F}$ type star and plotted in Fig. 2b. In the IUE SWP spectrum of Sp3 we do not find any evidence for the companion star spectrum. Since the F-type companion is several magnitudes fainter, its effect on the continuum flux of the central star in the SWP spectrum appears to be insignificant.

Assuming a temperature of $50000 \mathrm{~K}$ for the central stars, the integrated flux in the UV (1150 $\AA$ to $1950 \AA$ ) is $1.34 \times 10^{-9} \mathrm{erg} \mathrm{s}^{-1} \mathrm{~cm}^{-2}$ for $\mathrm{Hb} 7$ and $2.49 \times 10^{-9} \mathrm{erg} \mathrm{s}^{-1} \mathrm{~cm}^{-2}$ for Sp3. The integrated far infrared fluxes $(12 \mu$ to $100 \mu)$ for $\mathrm{Hb} 7$ and Sp3 with blackbody temperatures of $130 \mathrm{~K}$ and $100 \mathrm{~K}$ respectively are $0.59 \times 10^{-9} \mathrm{erg} \mathrm{s}^{-1} \mathrm{~cm}^{-2} 0.45 \times 10^{-9} \mathrm{erg} \mathrm{s}^{-1} \mathrm{~cm}^{-2}$. Thus, almost as much energy is radiated in the infrared as is seen coming from the central star(s).

In Table 1 we have listed the $B V I, J H K$ magnitudes of $\mathrm{Hb} 7$ and $\mathrm{Sp} 3$ adopted in this paper. The $J H K$ magnitudes for $\mathrm{Hb} 7$ were obtained from the 2MASS Point Source Catalog within a search radius of $6^{\prime \prime}$. The $K$ band image of $\mathrm{Hb} 7$ from the 2MASS Catalog is shown in Fig. 3. The NICMOS arrays mounted on the 2MASS telescopes provide a resolution of $2^{\prime \prime}$ per pixel. $\mathrm{Hb} 7$ is not resolved at this resolution and does not appear as an extended source in the 2MASS $J H K$ images.

\subsection{Dynamical age of the nebula}

For $\mathrm{Hb7}$, all distance estimates in the literature have been obtained assuming a nebular diameter of 4 " (VorontsovVelyaminov 1962). However, Vorontsov-Velyaminov's estimate of the nebular diameter was based on low resolution photographic plates and may be wrong. Recently, based on CCD images, Gorny et al. (1999) estimated an angular size of $13^{\prime \prime} \times 12^{\prime \prime}$ in $\mathrm{H}_{\alpha}$. We found this to be consistent with the nebular diameter estimated from the 2MASS images. Since distance estimates for $\mathrm{Hb} 7$ based on the wrong angular diameter of $4^{\prime \prime}$ cannot be used, we have used the relation between core-mass and quiescent luminosity maximum $\left(L_{\mathrm{Q}}\right)$ for AGB stars (Wood \& Zarro 1981) to derive the distance. Using $\mathrm{L}_{\mathrm{Q}}$ for the luminosity of the $\operatorname{star}\left(8828 L_{\odot}\right.$ for $\left.\mathrm{Hb} 7\right)$ and $M_{\text {bol }}(\operatorname{Sun})(=4.75)$ we found $M_{\mathrm{bol}}(\mathrm{Hb} 7)$ to be -5.1 . Applying the bolometric correction and using the formula for the distance modulus we obtained a distance of $5.5 \mathrm{kpc}$.

Gussie \& Taylor (1994) found two components in the expansion velocity distribution of a large sample of PNe.

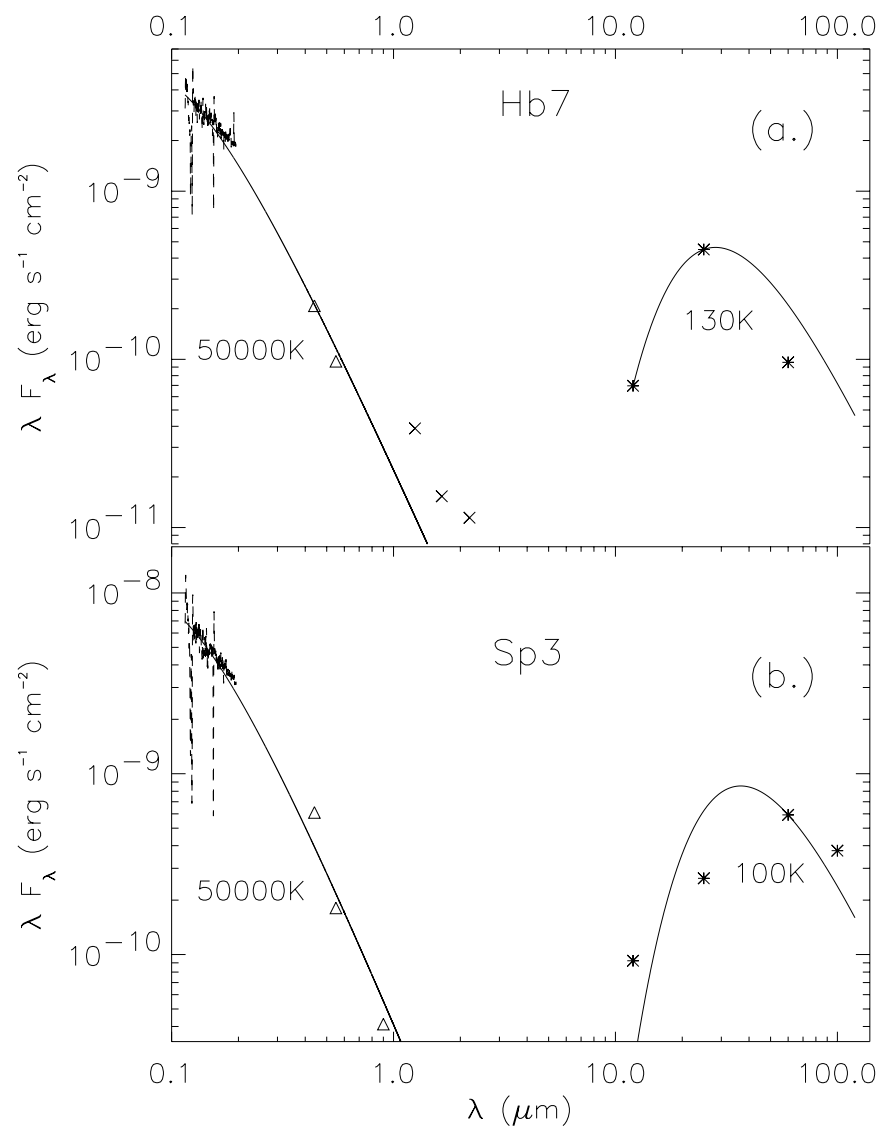

Fig. 2. Energy distribution of $\mathrm{Hb} 7$ a) and $\mathrm{Sp} 3 \mathrm{~b}$ ) from the UV to the far infrared is shown. The data is corrected for interstellar reddening using $E(B-V)=0.19$ for $\mathrm{Hb} 7$ and $E(B-V)=0.159$ for Sp3. IUE data (dashed line) is plotted along with $B V I$ (open triangles), JHK (crosses) and IRAS photometry (asterix marks).

Nebulae with the low-velocity component $\left(12.5 \mathrm{~km} \mathrm{~s}^{-1}\right)$ were found to be smaller in linear extent than highexpansion velocity nebulae $\left(27.5 \mathrm{~km} \mathrm{~s}^{-1}\right)$. Assuming an expansion velocity of $12.5 \mathrm{~km} \mathrm{~s}^{-1}$, angular diameter of $13^{\prime \prime}$ and distance of $5.5 \mathrm{kpc}$, we obtained a dynamical age of 13418 years for $\mathrm{Hb7}$.

Using Daub's (1982) formalism and an angular radius of $17.8^{\prime \prime}$ (Acker et al. 1992), Cahn et al. (1992) obtained a distance of $1.9 \pm 0.3 \mathrm{kpc}$ for Sp3. The angular diameter and expansion velocity of Sp3 is $35.5^{\prime \prime}$ and $22 \mathrm{~km} \mathrm{~s}^{-1}$ respectively (Acker et al. 1992). At a distance of $1.9 \mathrm{kpc}$, we found the age of the nebula to be 7278 years.

The theoretical evolutionary tracks of Blöcker \& Schönberner (1990) predict an age of 3000 years for PNe with $\log \left(T_{\text {eff }} / \mathrm{K}\right)$ of 4.7 and core mass of $0.605 M_{\odot}$, along 


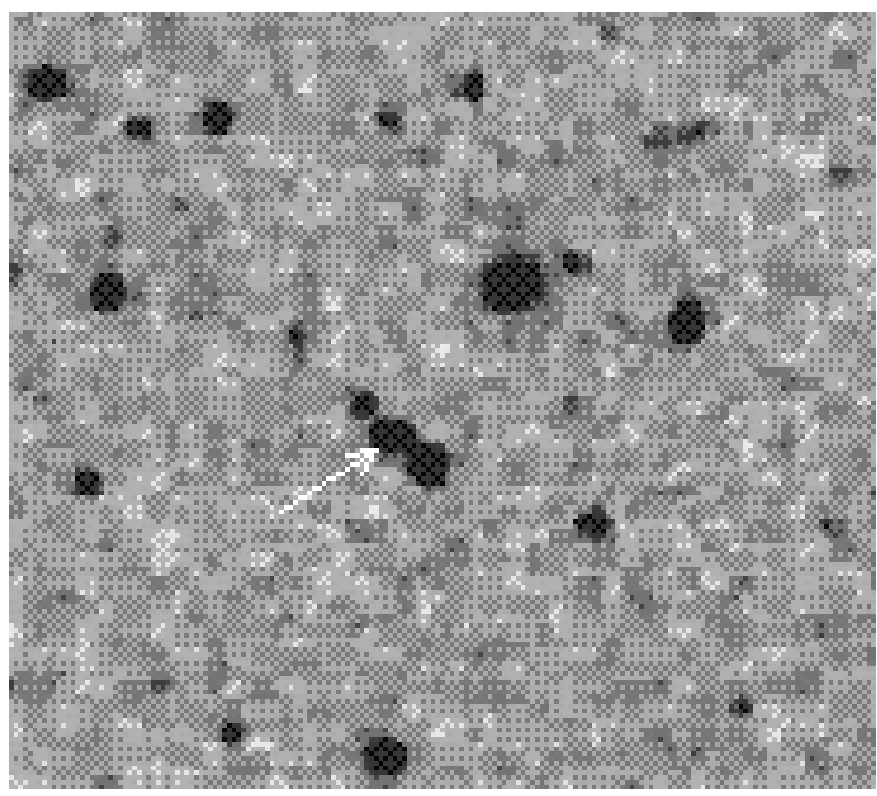

Fig. 3. 2MASS image of $\mathrm{Hb} 7$ in $K$ band.

the horizontal part of the evolutionary track on the HRdiagram.

\section{Discussion and conclusions}

Our analysis of the UV (IUE) spectra reveals that the central stars of $\mathrm{Hb} 7$ and $\mathrm{Sp} 3$ are O3 -dwarfs with effective temperatures of $50000 \mathrm{~K}$, core-mass of $0.644 M_{\odot}$ and $0.565 M_{\odot}$ and mass loss rates of $2.9 \times 10^{-8} M_{\odot} \mathrm{yr}^{-1}$ and $7 \times 10^{-9} M_{\odot} \mathrm{yr}^{-1}$ respectively. The IRAS fluxes of these objects revealed a cold dust component at $130 \mathrm{~K}$ for $\mathrm{Hb} 7$ and $100 \mathrm{~K}$ for Sp3. The cold dust component may be interpreted as thermal emission from the dust present in the circumstellar envelope of these stars, a remnant of the previous strong mass loss AGB phase. We estimated dynamical ages of $13.4 \times 10^{3} \mathrm{yrs}$ and $7.3 \times 10^{3} \mathrm{yrs}$ for $\mathrm{Hb} 7$ and Sp3 respectively.

Cerruti-Sola \& Perinotto (1985) investigated the frequency of occurence of stellar winds in CSPNe. They found that it depends on the stellar gravity in the sense that CSPNe with a gravity smaller than $\log g=5.2(\mathrm{cgs})$ almost always have a wind while at higher gravities the presence of wind becomes less and less frequent. The presence of wind in the CSPNe Hb7 and Sp3 indicates that their surface gravities $\log g<5.2$. Pauldrach et al. (1988) have shown that the presence of a fast wind in a CSPN depends not only on the stellar gravity but also on the luminosity. That is, the more a CSPN departs from the Eddington luminosity, the less frequent is the occurence of the wind.

Acknowledgements. MP is very thankful to Prof. S. Deguchi, Prof. K. Kodaira and Prof. N. Kaifu for their kind support and hospitality. We thank the referee for helpful comments.

\section{References}

Acker, A., Ochsenbein, F., Stenholm, B., et al. 1992, Strasbourg-ESO Catalogue of Galactic Planetary Nebulae Aller, L. H. 1976, PASP, 88, 574

Blöcker, T., \& Schönberner, D. 1990, A\&A, 240, L11

Cahn, J. H., Kaler, J. B., \& Stanghellini, L. 1992, A\&AS, 94, 399

Cerruti-Sola, M., \& Perinotto, M. 1985, ApJ, 291, 237

Ciardullo, R., Bond, H. E., Sipior, et al. 1999, ApJ, 118, 488

Daub, C. T. 1982, ApJ, 260, 612

Gorny, S. K., Schwarz, H. E., Corradi, R. L. M., \& Van Winckel, H. 1999, A\&A, 136, 145

Gussie, G. T., \& Taylor, A. R. 1994, PASP, 106, 500

Heap, S. R. 1986, in Eight Years of IUE, ESA SP-263, 291

Heck, A., Egret, D., Jaschek, M., \& Jaschek, C. 1984, IUE Low-Dispersion Spectra Reference Atlas - Part 1, Normal Stars, ESA SP-1052

Lang, K. R. 1992, Astrophysical Data: Planets and Stars (Springer-Verlag), 137

Parthasarathy, M., Garcia-Lario, P., Pottasch, S. R., et al. 1993, A\&A, 267, L19

Parthasarathy, M., Garcia-Lario, P., de Martino, D., et al. 1995, A\&A, 300, L25

Patriarchi, P., \& Perinotto, M. 1991, A\&AS, 91, 325

Pauldrach, A., Puls, J., Kudritzki, R. P., Mendez, R. H., \& Heap, S. R. 1988, A\&A, 207, 123

Preite-Martinez, A., Acker, A., Köppen, J., \& Stenholm, B. 1991, A\&AS,88, 12121

Prinja, R. K., Barlow, M. J., \& Howarth, I. D. 1990, ApJ, 361, 607

Prinja, R. K. 1994, A\&A, 289, 221

Samland, M., Köppen, J., Acker, A., \& Stenholm, B. 1992, A\&A, 264, 184

Savage, B. D., Massa, D., Meade, M., \& Wesselius, P. R. 1985, ApJS, 59, 397

Tylenda, R., Acker, A., Gleizes F., \& Stenholm B. 1989, A\&AS, 77,39

Tylenda, R., Acker, A., Stenholm, B., Gleizes, F., \& Raytchev, B. 1991, A\&AS, 89, 77

Vorontsov-Velyaminov, B. A. 1962, Soobse. Astr. Inst. Sternbergra, No. 118, 3

Wood, P. R., \& Zarro, D. M. 1981, ApJ, 247, 247 